\title{
El efecto del flipped classroom en la motivación por el aprendizaje del inglés como lengua extranjera en estudiantes de nivel pre-intermedio
}

\section{The effect of the flipped classroom method on pre-intermediate level students' motivation to learn English as a foreign language}

\author{
José Luis Ruiz Robles ${ }^{1}$ \\ ${ }^{1}$ Universidad del Atlántico - UA, Colombia \\ Correo a/Mail to: José Luis Ruiz Robles (joselruiz@mail.uniatlantico.edu.co)
}

Recibido/Submitted: 20 Oct. 2016; Aceptado/Approved: 13 Dic. 2016

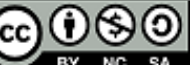

Copyright (C) 2016 Ruiz Robles, J. L.. Todo el contenido de la revista (incluyendo instrucciones, política editorial y modelos) está bajo una licencia Creative Commons Atribución-NoComercial-Compartirlgual 3.0 No Adaptada. Siendo publicados por esta revista, artículos son de libre uso en ambientes educativos, de investigación y no comerciales, con atribución de autoría obligatoria. Más información en http://revistas.ufpr.br/atoz/about/submissions\#copyrightNotice.

\begin{abstract}
Resumen
Introdución: el presente estudio tuvo como objetivo principal determinar la efectividad del flipped classroom para incrementar la motivación de los estudiantes en un curso de nivel pre-intermedio de inglés como lengua extranjera.

Método: a fin de alcanzar el objetivo trazado, un estudio cuasi-experimental de corte cuantitativo fue llevado a cabo. La población objeto de estudio para esta investigación estuvo conformada por 48 estudiantes de una escuela pública en la ciudad de Barranquilla, Colombia. Por medio de un muestreo no probabilístico, 25 de ellos compusieron el grupo control del estudio y 23 el grupo experimental. La variable independiente fue el uso del flipped classroom como estrategia instruccional, mientras que la dependiente fue el nivel de motivación por el aprendizaje del inglés como lengua extranjera, la cual fue medida a través de un escalamiento tipo Likert adaptado y previamente validado. Este instrumento fue administrado antes y después de la intervención.

Resultados: la información obtenida fue analizada con el soporte de un software de análisis cuantitativo y la definición de significancias estadísticas mediante el cálculo t de Student.

Conclusión: estos procesos permitieron concluir que el uso del flipped classroom aumentó la motivación por el aprendizaje del inglés como lengua extranjeras en la población objeto de estudio.
\end{abstract}

Palabras clave: Tecnologia; Inovación en educación; Procesos de enseñanza y aprendizaje; Idioma inglés; Inovación en el aprendizaje

\begin{abstract}
Introduction: the investigation arises from a research project that had the objective to determine the effectiveness of the flipped classroom as an instructional strategy to increase the pre-intermediate level students' motivation to learn English as a foreign language.

Method: in order to achieve this aim, a quasi-experimental study with quantitative techniques was conducted. The target population is composed of 48 students from a public school in the city of Barranquilla, Colombia. By means of a non-probabilistic sampling, 25 of them composed the control group and 23 of them the experimental group. The independent variable was the use of the flipped classroom as an instructional strategy while the dependent variable was the motivation level to learn English as a foreign language, which was measured via a previously adapted and validated Likert scale. This instrument was administrated before and after the intervention.

Results: the gathered information was analyzed through a quantitative analysis software and the definition of statistical significances with the t-Student calculus.

Conclusions: consequently, it is concluded that the use of the flipped classroom increased the motivation to learn English as a foreign language of the target population of the study.
\end{abstract}

Keywords: Technology; Innovation in education; Teaching and learning processes; English language; Innovation in learning

\section{INTRODUCCIÓN}

El mundo actual se caracteriza por la comunicación intercultural, por el creciente ritmo de los avances científicostecnológicos y por los procesos de internacionalización (Powell-Davies \& Otero, 2011). Estas circunstancias plantean la necesidad de un idioma común que le permita a la sociedad internacional acceder a este nuevo mundo globalizado (Crystal, 2003). En Colombia, la importancia del inglés se evidencia en la implementación de un prominente número de acuerdos multilaterales. "En el plano comercial, por ejemplo, se tienen acuerdos vigentes, suscritos o negociaciones en curso con una buena cantidad países o zonas económicas, tales como Estados Unidos" (Sánchez, 2013, p. 4).

Conscientes de lo anterior, en Colombia, por parte de instituciones gubernamentales y no gubernamentales, se han aunado esfuerzos significativos para fortalecer la enseñanza y aprendizaje del inglés como lengua extranjera en las instituciones del sistema educativo colombiano. No obstante, a pesar de estas reglamentaciones, Colombia es un país con un bajo nivel de bilingüismo entre la población estudiantil que egresa del sistema educativo, específicamente, con un bajo nivel de inglés. "La proporción de estudiantes bilingües o de aquellos que por lo menos superan el nivel pre-intermedio de inglés es muy reducida, mientras que la cantidad de estudiantes que 
no alcanza el nivel de principiante es significativamente alta” (Ruiz-Robles, 2014, p. 3). Esto se ratifica con los más recientes resultados de las pruebas de estado, en los cuales se pone de relieve que, en promedio, el 5\% de estudiantes que egresan de las escuelas del país tienen un nivel de proficiencia intermedio (Ministerio de Educación Nacional [MEN], 2015).

Así las cosas, se hace necesario la gestación de propuestas pedagógicas, iniciativas, inclusión de nuevos métodos, enfoques, entre otras estrategias a fin de fomentar la promoción de la enseñanza y el aprendizaje del inglés como lengua extranjera. Particularmente, el presente estudio abarca el diseño de una secuencia instruccional que emplea el flipped classroom, así como los recursos tecnológicos necesarios para la aplicación del mismo, analizando su impacto sobre la motivación de los participantes. Esta secuencia fue implementada durante dos bimestres lectivos en las sesiones de clases de inglés de nivel pre-intermedio con estudiantes próximos a egresar del sistema escolar. A continuación, se pone de relieve la pregunta problema del estudio y, consecuentemente, el objetivo del mismo:

Pregunta problema: ¿Qué efectividad tiene el flipped classroom para incrementar la motivación de los estudiantes de un curso de inglés pre-intermedio como lengua extranjera?

Objetivo de la investigación: Determinar la efectividad del flipped classroom para incrementar la motivación de los estudiantes de un curso de inglés pre-intermedio como lengua extranjera.

\section{FUNDAMENTACIÓN TEÓRICA}

\section{Motivación}

El término motivación deriva del verbo latino movere que significa moverse. Por consecuencia, la idea del movimiento está implícita en las ideas de sentido común sobre la motivación (Pintrich, Meece, \& Schunk, 2014). No obstante, actualmente, existe un vasto espectro de literatura especializada sobre la motivación en múltiples campos del conocimiento: psicología, educación, lingüística, marketing, etcétera. (Kleinginna \& Kleinginna, citados en Mayer, Roberts, \& Barsade, 2008), sin llegar a un consenso entre las múltiples ópticas de estudio, compilaron cientos de definiciones de motivación, concebidas desde las diferentes perspectivas o corrientes teóricas y ópticas de estudio.

Se han abordado, a lo largo del siglo pasado y del presente, diversas aproximaciones teóricas sobre la motivación. Desde la fisiológica, se afirma que la motivación se usa para "describir las fuerzas que actúan sobre un organismo o en su interior para que inicie y dirija la conducta” (Petri \& Govern, 2013, p. 16). Por su parte, desde la psicología, se preceptúa que "la motivación es un constructo teórico-hipotético que designa un proceso complejo que causa la conducta. En la motivación intervienen múltiples variables (biológicas y adquiridas) que influyen en la activación, direccionalidad, intensidad y coordinación del comportamiento encaminado a lograr determinadas metas" (Bisquerra, 2008, p. 165). En cuanto a la educación, se anota que la motivación es un estado interno que activa, dirige y mantiene el comportamiento de los estudiantes hacia la consecución de logros académicos y de aprendizaje (Woolfolk, 2010). Así pues, se puede apreciar que desde los diferentes enfoques, la motivación presenta elementos en común: activación, dirección y persistencia.

Dado la naturaleza del presente estudio, se profundiza en la teoría de motivación integradora de Gardner y Lambert (citados en Dörnyei \& Ushioda, 2009). En esta, se distingue una dicotomía motivacional: orientación instrumental y orientación integradora. Para Gardner y Lambert, el concepto de orientación instrumental hace alusión "al conjunto de motivos de índole práctica por los que un aprendiente estudia una nueva lengua" (Pérez-Ruiz, 2015, p. 3-4). En consecuencia, se orienta hacia objetivos pragmáticos concretos de aprendizaje (v.g. aprobar una prueba, obtener un título universitario o empleo, ascensos, alcanzar determinado nivel socio-económico, entre muchos otros). En contraste, la orientación integradora hace referencia a

una disposición positiva hacia la comunidad hablante con el deseo de interactuar, o el anhelo de asemejarse e identificarse con el modo de vida y los valores de la nueva cultura. Por lo tanto, los dos objetivos principales serían el deseo de comunicación o de integración con el grupo social de la cultura de la lengua meta. Esta orientación implica una empatía y un conjunto de sentimientos positivos hacia la nueva lengua y cultura (Pérez-Ruiz, 2015, p. 4).

\section{Flipped Classroom}

El término flipped classroom - expresión del idioma inglés que, literalmente, puede ser entendida como “dar la vuelta a la clase" o "una clase al revés" - fue acuñada por primera vez en el año 2000. No obstante, no fue sino hasta el 2007 que esta estrategia de instrucción tuvo implicaciones prácticas y el término fue consolidado en la comunidad americana (Bergmann \& Sams, 2012). Dos profesores del Instituto de Colorado en los Estados Unidos empezaron a grabar presentaciones en PowerPoint de los contenidos de sus clases con narraciones explicativas capturadas en vídeo con el objetivo de facilitarlas a los alumnos con dificultades para asistir a las sesiones de clase. "Para sorpresa de los docentes, estas grabaciones, en principio creadas para estudiantes no presenciales de sus clases, eran también seguidas por otros estudiantes” (Paz, Serna, Ramírez, Valencia, \& Reinoso, 2014, p. 397). Desde ese entonces, hasta la actualidad, casi una década después, estas prácticas siguen 
replicándose a lo largo de las escuelas del mundo, especialmente las de Estados Unidos, Australia y el centro de Europa (Herreid \& Schiller, 2013).

Hoy día, el flipped classroom se ha consolidado como una estrategia instruccional y, además, una modalidad de aprendizaje semi-presencial. El objetivo principal de la misma se centra en invertir la forma en que los contenidos son entregados para dar mayor tiempo a la práctica, ejercitación, transferencia, construcción y aplicación de teorías y conceptos. Los contenidos son revisados en casa, mediante la reproducción de objetos virtuales de aprendizaje por los estudiantes y la práctica es realizada en clase. Esta perspectiva, dentro de los procesos de enseñanza y aprendizaje, es considerada por Bergmann y Sams (2012), como una metodología y/o perspectiva pedagógica que propone un modelo de aprendizaje caracterizado por el rol activo del aprendiz.

El potencial de esta perspectiva radica en que el tiempo invertido en explicar la materia de manera magistral, queda relegado al trabajo que el estudiante realiza tranquilamente en casa a través de la explicación estratégica en grabaciones de vídeo. Dichos materiales son estudiados por los estudiantes en su casa, con la ventaja de que pueden hacerlo cuantas veces considere necesario. Las tareas tradicionales que el docente explica en clase y que luego son realizadas por el estudiante en casa, pueden ser realizadas en el salón de clase con el beneficio que esto tiene para el estudiante, debido a que las dudas, opiniones, y resoluciones de las mismas se pueden llevar a cabo mediante la interacción entre compañeros y docente, posibilidad que no se presenta al realizar este tipo de actividades en casa (Paz et al., 2014, p. 397).

De otra parte, cabe anotar que múltiples estudios (Bergmann \& Sams, 2012; Berrett, 2012; Brunsell \& Horejsi, 2011; Fulton, 2012; Ronchetti, 2010) sugieren que métodos instruccionales como flipped classroom, los cuales soportan el aprendizaje activo, beneficia a los estudiantes más que el modelo de clase tradicional basada en la lectura y compromisos escolares en casa. Entre estos beneficios, se encuentra documentando el impacto positivo en la motivación por el aprendizaje. Esto se debe, en ilación con Touchton (2015), a que los estudiantes, en el marco del aula invertida, ejecutan las tareas de menos exigencia cognitiva afuera del aula de clases, mientras que las asignaciones con más carga cognitiva - atendiendo a la taxonomía de Bloom (citado en Touchton, 2015) - se llevan a cabo dentro de esta, con el soporte del docente e intervenciones significativas.

Entre las más notables características del flipped classroom - en consonancia con Brunsell y Horejsi (2011) - se anotan las siguientes:

a) Los objetos virtuales de aprendizaje (v.g. vídeos, esquemas, libros electrónicos, pistas de audio, software educativo, etcétera) sustituyen al docente en la instrucción directa.

b) Fomenta la interacción y el tiempo de contacto personalizado entre alumnos y profesores.

c) El contenido curricular queda archivado en los repositorios o plataformas de enseñanza institucionales que, eventualmente, puede ser revisado, supervisado o reparado.

d) Los estudiantes son responsables de su propio aprendizaje.

e) Se lleva a cabo una combinación entre la instrucción directa y el aprendizaje constructivista.

f) Los alumnos están involucrados plenamente en su propio aprendizaje.

A fin de construir o seleccionar los objetos virtuales de aprendizaje necesarios para soportar las secuencias de enseñanza-aprendizaje, los docentes y diseñadores curriculares, actualmente, cuentan con amplio catálogo de herramientas para la elaboración de recursos educativos digitales abiertos. Como se plasma en la Cuadro 1, los formatos más comunes de estos recursos son presentaciones interactivas, vídeos, pistas de audio, documentos, documentos con texto enriquecido, gráficos y esquemas conceptuales.

Luego de la selección o diseño de objetos virtuales de aprendizaje para el soporte de las secuencias didácticas, el docente debe seleccionar una plataforma para su difusión. Así, los estudiantes tienen acceso al contenido dispuesto e inician con la ejecución o reproducción del mismo desde sus hogares para su posterior reflexión, aplicación y trasferencia en el aula de clase, a través de actividades significativas. Como se pone de relieve en la Cuadro 2, las principales herramientas para la difusión de estos recursos son Moodle, Edmodo, blogs, páginas web, wikis y redes sociales como Twitter y Facebook.

\section{METODOLOGÍA}

\section{Diseño}

Dada la naturaleza del presente estudio, este se enmarca en el enfoque cuantitativo de la investigación científica, de alcance correlacional y con un diseño de tipo experimental, específicamente, cuasiexperimental (Hernández, Fernández, \& Baptista, 2014).

En cuanto al enfoque cuantitativo, el proceso de investigación de este estudio - como se esquematiza en la Figura 1- inicia con la formulación de una serie hipótesis, previo a la inmersión inicial y profunda en el campo objeto de estudio. Posteriormente, estas hipótesis son probadas para su verificación a través de la experimentación estructurada, en este caso, a través de la aplicación del flipped classroom durante las sesiones de inglés en un grupo experimental, mientras en un grupo control se llevan a cabo sesiones de clase según el currículo 


\begin{tabular}{|c|c|c|}
\hline Formato & Herramientas & Características \\
\hline \multirow[t]{4}{*}{ Presentaciones } & Microsoft PowerPoint & $\begin{array}{l}\text { Software para la elaboración de presentaciones en diapositivas con gran re- } \\
\text { conocimiento y múltiples herramientas de edición, nativo del sistema operativo } \\
\text { Windows. }\end{array}$ \\
\hline & Keynote & $\begin{array}{l}\text { Software nativo y de uso exclusivo en el sistema operativo Macintosh para la elabo- } \\
\text { ración de presentaciones en diapositivas. Cuenta con una variedad de plantillas y } \\
\text { herramientas de enriquecimiento estético y gráfico. Se debe adquirir una licencia } \\
\text { paga para su uso. }\end{array}$ \\
\hline & Prezi & $\begin{array}{l}\text { Software y aplicativo web para el diseño de presentaciones interactivas que pueden } \\
\text { ser visualizadas en internet, o bien, mediante un archivo en formato flash. Cuenta } \\
\text { con una versión básica para uso gratuito. }\end{array}$ \\
\hline & Slideshare, Scribd & $\begin{array}{l}\text { Plataformas web para la creación de presentaciones, publicación de documentos y } \\
\text { difusión de los mismos empleando código HTML que puede ser embebido en páginas } \\
\text { web, wikis y blogs. }\end{array}$ \\
\hline \multirow{6}{*}{ Vídeos y pistas de audio } & Windows Movie Maker & $\begin{array}{l}\text { Editor de vídeos nativo y de uso exclusivo para el sistema operativo Windows. } \\
\text { Cuenta con herramientas básicas para la creación de vídeos basados en secuen- } \\
\text { cias de imágenes. }\end{array}$ \\
\hline & $\begin{array}{l}\text { Powtoon, Go-animate!, } \\
\text { Animoto }\end{array}$ & $\begin{array}{l}\text { Plataformas web para la creación de vídeos basados en secuencias sincrónicas de } \\
\text { imagen, texto, transiciones, animaciones y sonidos. Cuentan con versiones gratuitas } \\
\text { y de uso limitado. }\end{array}$ \\
\hline & YouTube & Plataforma para la creación, edición y difusión de vídeos de diversa índole. \\
\hline & Jing & $\begin{array}{l}\text { Software freeware para la realización de objetos de aprendizaje basados en screen- } \\
\text { cast o grabación de pantalla, en conjunto con otros periféricos (v.g. micrófono, } \\
\text { cámara). }\end{array}$ \\
\hline & Adobe Presenter & $\begin{array}{l}\text { Software con licencia paga para el soporte de instrucción a distancia basado en } \\
\text { screencast o grabación de pantalla con una integración de diferentes soluciones } \\
\text { ofimáticas (v.g. Microsoft PowerPoint). Disponible para Windows y Macintosh. }\end{array}$ \\
\hline & Soundcloud, Ivoox & Plataforma para la creación y difusión de podcasts. \\
\hline \multirow{5}{*}{$\begin{array}{l}\text { Documentos, documen- } \\
\text { tos con texto enrique- } \\
\text { cido, gráficos y esquemas } \\
\text { conceptuales }\end{array}$} & Microsoft Publisher & $\begin{array}{l}\text { Software con licencia de pago para la elaboración de una amplia gama de documen- } \\
\text { tos impresos y digitales que hace hincapié en el diseño y la maquetación. Disponible } \\
\text { para Windows y Macintosh. }\end{array}$ \\
\hline & Pages & $\begin{array}{l}\text { Software con licencia de pago, nativo y de uso exclusivo de Macintosh, para la elabo- } \\
\text { ración de una amplia gama de documentos impresos y digitales. }\end{array}$ \\
\hline & Microsoft Word & $\begin{array}{l}\text { Software editor de textos con licencia de pago. Disponible para los sistemas opera- } \\
\text { tivos Windows y Macintosh. }\end{array}$ \\
\hline & Mindomo & $\begin{array}{l}\text { Plataforma web para la creación de mapas de pensamiento interactivos. Cuenta con } \\
\text { versiones básicas de uso gratuito. }\end{array}$ \\
\hline & Cmaps & $\begin{array}{l}\text { Software de licencia abierta para el diseño de mapas de pensamiento disponible } \\
\text { para Windows. }\end{array}$ \\
\hline
\end{tabular}

Cuadro 1. Principales herramientas para la creación o selección de objetos virtuales de aprendizaje. Fuente: los autores.

\begin{tabular}{|c|c|c|}
\hline Herramienta & Características & Acceso a información adicional \\
\hline Moodle & $\begin{array}{l}\text { Software para la creación y gestión de cursos en línea. Con- } \\
\text { templa el desarrollo de un entorno virtual de aprendizaje } \\
\text { que engloba actividades de comunicación, comprensión y } \\
\text { evaluación. Cuenta con una vasta gama de herramientas } \\
\text { tanto para docentes como para estudiantes que facilitan } \\
\text { los procesos de enseñanza-aprendizaje. }\end{array}$ & https://docs.moodle.org/all/ \\
\hline Edmodo & $\begin{array}{l}\text { Plataforma educativa gratuita con características similares } \\
\text { a la de una red social que permite la creación de grupos } \\
\text { cerrados entre alumnos y profesores con el objetivo de } \\
\text { compartir mensajes, enlaces, documentos, eventos, prue- } \\
\text { bas, entre otros. }\end{array}$ & https://edmodo.com/about/ \\
\hline Blogs y páginas web & $\begin{array}{l}\text { Espacio web que permite la difusión de material multime- } \\
\text { dia de forma cronológica y permite a los alumnos comentar } \\
\text { las entradas y los comentarios de sus iguales. }\end{array}$ & $\begin{array}{l}\text { https://wordpress.com/about/ } \\
\text { https://blogger.com/about/ } \\
\text { https://tumblr.com/about/ } \\
\text { https://edublogs.org/features/ }\end{array}$ \\
\hline Wikis & $\begin{array}{l}\text { A diferencia del blog, los wikis son colecciones concate- } \\
\text { nadas de páginas de internet que pueden ser creadas, edi- } \\
\text { tadas o eliminadas por todos los usuarios. }\end{array}$ & http://wikispaces.com/content/classroom/about/ \\
\hline Twitter y Facebook & $\begin{array}{l}\text { Redes sociales abiertas que posibilitan la creación de gru- } \\
\text { pos privados y públicos, así como listas de difusión para la } \\
\text { publicación de contenido multimedia. Asimismo, permite } \\
\text { a los alumnos comentar las entradas y los comentarios de } \\
\text { sus iguales. }\end{array}$ & $\begin{array}{l}\text { https://facebook.com/ } \\
\text { https://twitter.com/ }\end{array}$ \\
\hline
\end{tabular}

Cuadro 2. Principales herramientas para la difusión de los objetos virtuales de aprendizaje. Fuente: los autores.

institucional. Este proceso de prueba sistemática envuelve un mecanismo estadístico a fin de determinar relaciones entre las variables. Con el objetivo de medir estas variables, se adapta un escalamiento tipo Likert que mide los niveles motivacionales de los estudiantes antes y después de la intervención experimental. En cuanto al diseño cuasiexperimental, se anota que se manipula deliberadamente una variable independiente (el flipped classroom como método instruccional) para observar su efecto y relación con la variable dependiente 
(motivación por el aprendizaje del inglés).

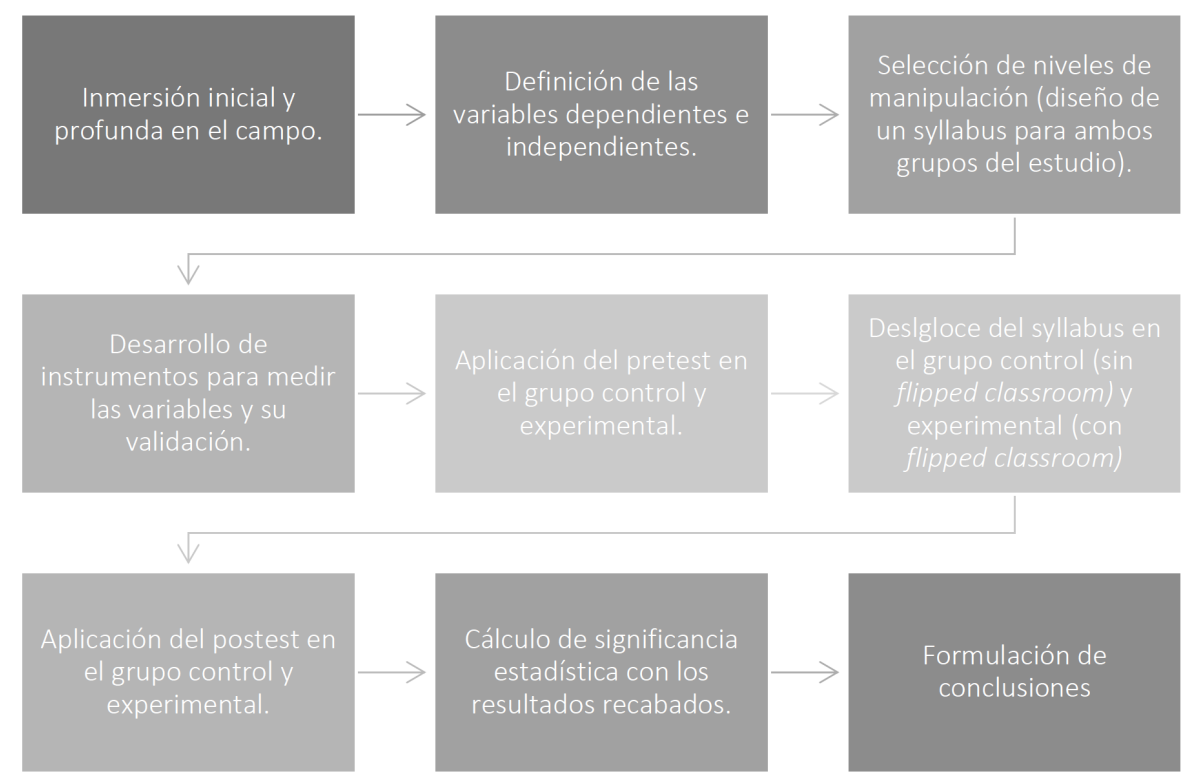

Figura 1. Procesos secuenciales de la investigación.

Fuente: los autores.

\section{Variables}

Variable dependiente: motivación por el aprendizaje del inglés como lengua extranjera. En esta variable se distinguen dos constructos: la orientación integradora y la orientación instrumental.

Variable independiente: uso del flipped classroom como estrategia instruccional durante las sesiones de clase de inglés. La manipulación de la variable dependiente, mediante el uso del flipped classroom, se llevó a cabo mediante el diseño y aplicación de una propuesta de intervención (véase Cuadro 3).

\begin{tabular}{|l|l|}
\hline \multicolumn{2}{|c|}{ Componentes instruccionales para el grupo control y experimental } \\
\hline Curso formativo & Inglés como lengua extranjera (EFL). \\
\hline Nivel de proficiencia & $\begin{array}{l}\text { Pre-intermedio atendiendo al Marco común europeo de referencias para las lenguas } \\
\text { (MCERL). }\end{array}$ \\
\hline Intensidad horaria/duración de la intervención & Cuatro meses con una intensidad horaria de cuatro horas semanales. \\
\hline Competencias & Competencia comunicativa: lingüística, sociolingüística y pragmática. \\
\hline Habilidades de comprensión y producción & Lectura, escritura, escucha y habla. \\
\hline Contenidos y logros instruccionales. & $\begin{array}{l}\text { Alineados a los Estándares básicos de competencia del Ministerio de Educación Na- } \\
\text { cional [MEN] (2006). }\end{array}$ \\
\hline Estrategias de evaluación & $\begin{array}{l}\text { Portafolios, rúbricas, matrices, evaluación por pares, autoevaluación, evaluación } \\
\text { sumativa mediante test estandarizados de proficiencia. }\end{array}$ \\
\hline Recursos educativos & $\begin{array}{l}\text { Computadores, conexión de banda ancha, diademas, altavoces, proyectores, recur- } \\
\text { sos basados en software (procesadores de texto, presentaciones en diapositivas, } \\
\text { navegador web, reproductor de multimedia, etc.). }\end{array}$ \\
\hline \multirow{2}{*}{ Metodología } & $\begin{array}{l}\text { En el grupo control se emplea una método instruccional de corte tradicional, en } \\
\text { consonancia a los preceptos institucionales. En el aula, se hace la revisión teórica, de } \\
\text { forma magistral de los patrones gramaticales. En casa, se le asignan a los estudiantes } \\
\text { tareas de investigación, creación y aplicación. }\end{array}$ \\
\cline { 2 - 2 } & $\begin{array}{l}\text { En el grupo experimental se realiza una concatenación de las actividades de apren- } \\
\text { dizaje atendiendo la taxonomía de Bloom (citado en Marzano \& Kendall, 2006). Así } \\
\text { pues, en casa, se llevan a cabo procesos de comprensión y retención de la infor- } \\
\text { mación. En contraste, en la clase, se dan procesos de creación, evaluación, análisis } \\
\text { y aplicación de los conceptos y constructos comprendidos en casa, mediante la } \\
\text { mediación docente. }\end{array}$ \\
\hline
\end{tabular}

Cuadro 3. Propuesta de intervención.

Fuente: los autores.

\section{Hipótesis}

H0. No hay una diferencia estadísticamente significativa entre el nivel de motivación por el aprendizaje del inglés como lengua extranjera antes y después de la implementación del flipped classroom.

H1. Hay una diferencia estadísticamente significativa (positiva) entre el nivel de motivación por el aprendizaje del inglés como lengua extranjera antes y después de la implementación del flipped classroom. 
H2. Hay una diferencia estadísticamente significativa (negativa) entre el nivel de motivación por el aprendizaje del inglés como lengua extranjera antes y después de la implementación del flipped classroom.

\section{Población y muestra}

La población objeto de estudio consta de 68 estudiantes, distribuidos en tres aulas de clase, que cursan undécimo grado en una institución educativa pública en Barranquilla - ciudad ubicada en la costa norte de Colombia. Así pues, los participantes, durante el estudio de experimentación, se encuentran próximos a egresar del sistema escolar. Estos tienen edades que oscilan entre los 15 a 17 años y cuentan con condiciones socio-económicas modestas. Cabe resaltar que los involucrados en este estudio asisten a sesiones de clases de inglés integradas en el currículo durante cuatro horas a la semana y su nivel de proficiencia es pre-intermedio.

Con respecto a la muestra, teniendo en cuenta la naturaleza de este estudio, se lleva a cabo un proceso de muestreo no probabilístico - en consonancia con los presupuestos de Hernández et al. (2014). Una porción (25 estudiantes de undécimo grado de un aula de clases específica) de la población total compone el grupo control del estudio y otra porción (23 estudiantes de undécimo grado de otra aula de clases) componen el grupo experimental, para un total de 48 participantes. De acuerdo con Hernández et al. (2014) y considerando los diseños cuasiexperimentales, este tipo de estudio deben ser llevados a cabo con un mínimo de 15 individuos. Así pues, el presente estudio cumple con este criterio de validez para el diseño de investigación acogido.

\section{Instrumentos de recolección de la información}

A fin de medir la variable dependiente del estudio, se adaptó y se tradujo al español el cuestionario Attitude/Motivation Test Battery de Gardner (2004) para el International AMTB Research Project, incluyendo algunos enriquecimientos lingüísticos (véase Apéndice A). Gardner (2004) pone de relieve una serie de instrumentos de recolección de la información para medir la motivación y los componentes asociados a esta: la orientación integradora y la integración instrumental. Entre estos instrumentos, Gardner diseña escalamientos tipo Likert y diferenciales semánticos fácilmente adaptables a un vasto número de contextos y poblaciones objeto de estudio. Dado los objetivos del presente estudio, se seleccionó el escalamiento tipo Likert (Gardner, 2004) que consta de 25 afirmaciones relacionadas con la motivación por el aprendizaje del inglés. Cada afirmación es evaluada por los participantes con cinco opciones de respuesta: totalmente de acuerdo, de acuerdo, ni en acuerdo ni en desacuerdo, en desacuerdo y muy en desacuerdo. El cuestionario tiene un puntaje final mínimo de 25 y máximo de 125.

En cuanto a la validación, cabe resaltar que la Attitude/Motivation Test Battery ha sido aplicada y validada en un vasto número de investigaciones sobre la motivación de los estudiantes de sexto a undécimo grado, en cursos de lengua nativa, segunda lengua y lengua extranjera (Dörnyei, 2001; Gardner, 2004). Los coeficientes de Cronbach $\alpha$ para los ítems que conforman el cuestionario fueron superiores a un valor de 0.70; la consistencia interna del instrumento es sustancial, luego de su administración en la prueba piloto. Cabe anotar que Dörnyei (2007) plantea que un valor de Cronbach $\alpha$ entre .60 y .70 son suficientes en el campo de lingüística aplicada. De otra parte, Gardner (1985) presenta información considerablemente relevante de la validez convergente y discriminante de las escalas e ítems.

\section{Instrumentos de análisis de la información}

Durante el proceso de análisis de los datos, se utiliza el software Statistical Package for the Social Sciences -SPSS - de IBM versión 23 para Macintosh, a fin de tabular los resultados proporcionados por el escalamiento tipo Likert y emplear el cálculo $t$ de Student. De este modo, se determina la significancia estadística entre los resultados de los test que tuvieron lugar antes y después de la intervención en los grupos control y experimental, dando lugar a la aceptación o rechazo de las hipótesis del estudio. Asimismo, por medio de este software estadístico, se ahonda en los promedios, desviaciones estándar y porcentajes de cada una de las categorías de análisis. Además de esto, se emplea Microsoft Office Visio y Microsoft Office Excel para representar los datos en gráficas de distribución y tendencias, así como también Microsoft Office Word para las tablas de información correspondientes.

\section{RESULTADOS Y ANÁLISIS DE DATOS}

A continuación, se ponen de relieve los resultados recabados y su análisis estadístico correspondiente. En el Apéndice B se encuentran los puntajes de los participantes en el Attitude/Motivation Test Battery antes y después de la intervención.

De otra parte, se anota que las puntuaciones de los escalamientos tipo Likert antes de la intervención eran de media similares y aproximadamente normales. La prueba ANOVA, ejecutada en el software SPSS, no mostró una diferencia estadísticamente significativa. Asimismo, la prueba de Levene para la homogeneidad de varianzas 
confirmó que se podía asumir la igualdad de varianzas para los resultados de los instrumentos antes de la intervención. Finalmente, las desviaciones de la normalidad no afectan la validez del análisis.

Ahondando en el análisis de los datos, en el grupo control - como puede apreciarse en la Tabla 1 y Figura 2-, se obtuvo un promedio de 45.64 de 125 puntos posibles antes de la experimentación y, posterior a esta, 46.40, en el instrumento de recolección. En consecuencia, y considerando el análisis de significancia estadística (véase Tabla 2), en el grupo control no hubo una diferencia estadísticamente significativa en los promedios del cuestionario de motivación por el aprendizaje del inglés. Esto era de esperarse, puesto que, en el grupo control, no hubo ningún tipo de intervención; las sesiones de clase fueron llevadas a cabo de forma usual, siguiendo los preceptos del currículo institucional.

\begin{tabular}{lccccc}
\hline & \multicolumn{2}{c}{ Grupo control } & & \multicolumn{2}{c}{ Grupo experimental } \\
\cline { 2 - 3 } \cline { 5 - 6 } & Pretest & Postest & & Pretest & Postest \\
\hline Promedio & 45.64 & 46.40 & & 43.00 & 66.00 \\
Desviación estándar & 17.00 & 17.02 & & 13.61 & 17.95 \\
Muestra & 25 & 25 & & 23 & 23 \\
\hline
\end{tabular}

Tabla 1. Contraste de los promedios y desviaciones estándar de los puntajes obtenidos en el pre y pos test en el grupo control y experimental. Fuente: los autores.

En cuanto al grupo experimental - como se esboza en la Tabla 1 y Figura 2-, se señala que este tuvo un promedio de 43 de 125 puntos posibles, frente a 45.6 del grupo control durante el pretest. En contraste, luego de la intervención mediante la aplicación del flipped classroom los resultados del postest alcanzaron, en promedio, 66 puntos en el grupo experimental. Por consiguiente, como se devela en el análisis de significancia estadística - plasmado en la Tabla 2-, en el grupo experimental, la diferencia entre los puntajes del Attitude/Motivation Test Battery antes y después de la intervención son altamente significativos, ascendiendo el valor $p$ a -0.0001 .

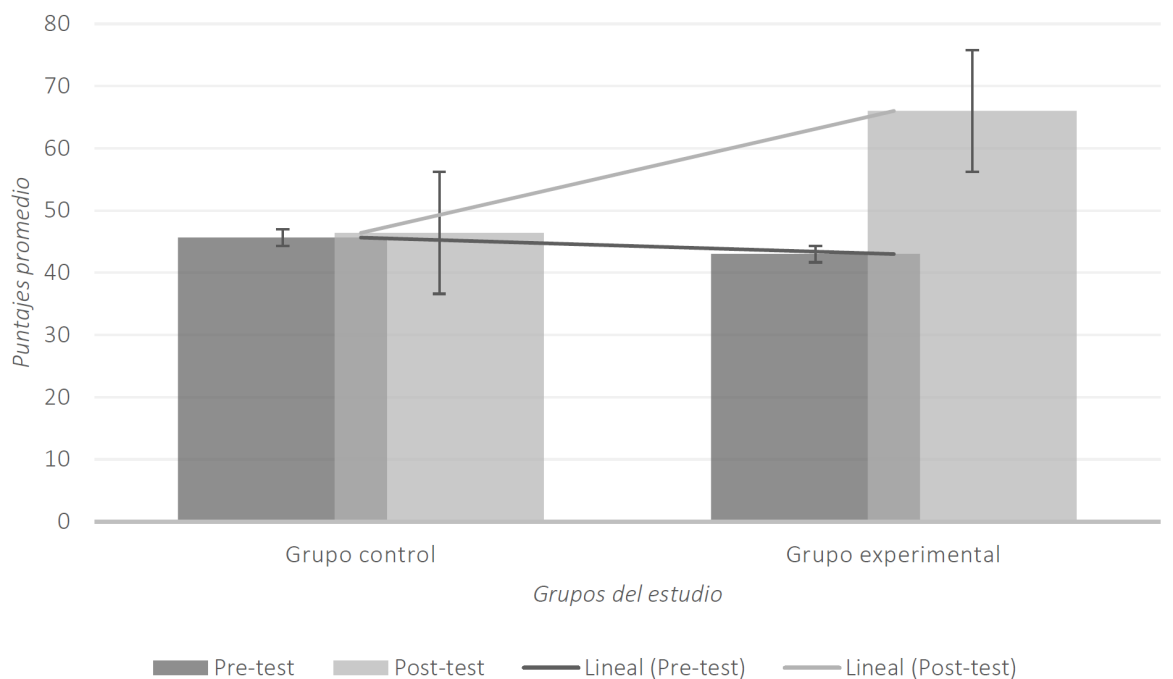

Figura 2. Tendencias en los resultados del Attitude/Motivation Test Battery en los grupos control y experimental. Fuente: los autores.

\begin{tabular}{lcc}
\hline & Grupo control & Grupo experimental \\
\hline Valor $p$ & -0.76 & -0.0001 \\
\hline Valor $t$ & 0.1580 & 4.8966 \\
\hline Grados de libertad $d f$ & 48 & 44 \\
\hline Error estándar de diferencia & 4.811 & 4.697 \\
\hline $\begin{array}{l}\text { ¿Hay una diferencia estadísticamente sig- } \\
\text { nificativa entre el pretest y postest? }\end{array}$ & No & Sí \\
\hline Muestra & 25 & 23 \\
\hline
\end{tabular}

Tabla 2. Análisis de significancia estadística.

Fuente: los autores.

Así las cosas, la primera hipótesis de la investigación - hay una diferencia estadísticamente significativa (positiva) entre el nivel de motivación por el aprendizaje del inglés como lengua extranjera antes y después de la implementación del flipped classroom - es aceptada. A su vez, la hipótesis nula es rechazada. A tenor de lo anterior, cabe remarcar el papel del uso del flipped classroom como estrategia instruccional en el incremento de la motivación de los estudiantes durante las sesiones de clase de inglés. 


\section{CONCLUSIONES}

Luego de la experimentación mediante el uso del flipped classroom durante las sesiones de clase de inglés como lengua extranjera, la correspondiente administración de instrumentos de recolección de la información, recabado de datos y su volcamiento en el software de análisis estadístico, se concluye lo siguiente:

a) El uso del flipped classroom - aula invertida - como estrategia instruccional previamente diseñada, teniendo en cuenta los múltiples factores condicionantes del contexto, permite aumentar la motivación por el aprendizaje del inglés de forma significativa y sus componentes; esto es, orientación instrumental y orientación integradora de forma significativa. Estos hallazgos constatan investigaciones anteriores sobre las implicaciones pedagógicas del flipped classroom (Bergmann \& Sams, 2012; Berrett, 2012; Brunsell \& Horejsi, 2011; Fulton, 2012; Ronchetti, 2010).

b) Además de las implicaciones en los niveles motivacionales de los estudiantes, se pudo determinar que el logro académico de los mismos aumentó considerablemente.

c) Se alcanzó todos los objetivos instruccionales trazados para el curso, alcanzando el nivel de proficiencia de la lengua contemplado por el currículo institucional. En contraste, en el grupo control esto no sucedió. Así pues, el uso del flipped classroom, no solo tiene efectos positivos en la motivación, sino también en el incremento del logro académico y niveles de proficiencia.

d) La creación de objetos de aprendizaje, considerando las necesidades educativas de los estudiantes, en el marco de los recursos educativos digitales abiertos, se configura como una apuesta de gran preponderancia, debido a la exponencial acogida de este tipo de recursos en la comunidad educativa internacional.

e) Cabe anotar que, para lograr la implementación un programa formativo, empleando la estrategia de instrucción del flipped classroom, se hace indispensable que los estudiantes y docentes cuenten con las competencias y habilidades necesarias para el uso apropiado de las herramientas tecnológicas. Asimismo, es de vital importancia contar con los recursos tecnológicos adecuados en el aula de clase para la mediación de las actividades, así como también los dispositivos y periféricos necesarios en las casas de los estudiantes. Así pues, se deben reunir ciertas características de acceso a este tipo de recursos. En poblaciones educativas donde existe una determinada brecha digital, este tipo de estrategias constituye un gran reto a la hora de su implementación. No obstante, con la conjugación de esfuerzos por parte de los diferentes estamentos educativos, podrían llevarse a cabo pruebas piloto para la posterior aplicación sistemática del flipped classroom.

f) En cuanto las líneas de continuidad para futuras investigaciones, se propone el estudio de los efectos del flipped classroom como estrategia instruccional en otras variables de estudio, tales como ambiente escolar, logro académico (nivel de proficiencia), motivación de logro y teorías motivacionales de corte metacognitivo. 


\section{REFERENCIAS}

Bergmann, J., \& Sams, A. (2012). Flip your classroom reach every student in every class every day. Alexandria: ASCD-ISTE.

Berrett, D. (2012). How 'Flipping' the classroom can improve the traditional lecture. Chronicle of Higher Education, 12, 114. Descargado de http://www.chronicle.com/article/How -Flipping-the-Classroom/130857/

Bisquerra, A. (2008). Educación emocional y bienestar. Barcelona: Wolters Klwwer.

Brunsell, E., \& Horejsi, M. (2011). Science 2.0: "Flipping" your classroom. The Science Teacher, 78(2), 10. Descargado de http://www.jstor.org/stable/24148320

Crystal, D. (2003). English as a global language (2a ed.). New York: Cambridge University.

Dörnyei, Z. (2001). New themes and approaches in second language motivation research. Annual Review of Applied Linguistics, 21, 43-59. doi: 10.1017/S0267190501000034

Dörnyei, Z. (2007). Research methods in applied linguistics: quantitative, qualitative, and mixed methodologies. Oxford: Oxford University.

Dörnyei, Z., \& Ushioda, E. (2009). Motivation, language identities and the L2 self: A theoretical overview. En Z. Dörnyei \& E. Ushioda (Eds.), Motivation, language identity and the L2 self. Bristol/New York/Canadá: Multilingual Matters.

Fulton, K. (2012, June/July). Upside down and inside out: Flip your classroom to improve student learning. Learning \& Leading with Technology, 12-17. Descargado de http://www.learningandleading-digital.com/ learning_leading/20120607?pg=14\#pg14

Gardner, R. C. (1985). Social psychology and second language learning: the role of attitudes and motivation. London: Edward Arnold.

Gardner, R. C. (2004). Attitude motivation test battery: International AMTB research project. Ontario: The University of Western Ontario.

Hernández, R., Fernández, C., \& Baptista, M. (2014). Metodología de la investigación (6a ed.). México: McGrawHill.

Herreid, C. F., \& Schiller, N. (2013). Case study: Case studies and the flipped classroom. Journal of College Science Teaching, 42(5). Descargado de http://www.nsta.org/store/product _detail.aspx?id=10.2505/4/jcst13_042_05_62

Marzano, R. J., \& Kendall, J. S. (2006). The new taxonomy of educational objectives. Thousand Oaks: Corwin Press.

Mayer, J. D., Roberts, R. D., \& Barsade, S. G. (2008). Human abilities: Emotional intelligence. Annual Review of Psychology, 59, 507-536. doi: 10.1146/annurev.psych.59.103006.093646

Ministerio de Educación Nacional. (2006). Estándares básicos de competencias en lenguas extranjeras: Inglés. Bogotá.

Ministerio de Educación Nacional. (2015). Programa nacional de inglés 2015-2025: Documento de socialización. Bogotá.

Paz, A. P., Serna, A., Ramírez, M. I., Valencia, T., \& Reinoso, J. (2014). Hacia la perspectiva de aula invertida (Flipped Classroom) en la Pontificia Universidad Javeriana desde una tipología de uso educativo del Sistema Lecture Capture (S.L.C). En Novena conferencia latinoamericana de objetos y tecnologías de aprendizaje (pp. 395-403). Manizales, Colombia. Descargado de http://www.laclo.org/papers/index.php/ laclo/article/view/265/247
Petri, H., \& Govern, J. (2013). Motivation: Theory, research, and application (6a ed.). New York: Thomson-Wadsworth.

Pintrich, P., Meece, J., \& Schunk, D. (2014). Motivation in education: Theory, research, and applications (4a ed.). New York: Pearson.

Powell-Davies, P., \& Otero, J. (2011). Word for word: The social, economic and political impact of spanish and english. Madrid: British Council; Instituto Cervantes.

Pérez-Ruiz, J. (2015). Actitudes sociolingüísticas en estudiantes universitarios taiwaneses de ELE. Marco ELE, 21, 1-18. Descargado de http://marcoele.com/actitudes -sociolinguisticas-en-estudiantes-taiwaneses-de-ele/

Ronchetti, M. (2010). Using video lectures to make teaching more interactive. International Journal of Emerging Technologies in Learning, 5(2), 45-48. doi: 10.3991/ijet.v5i2.1156

Ruiz-Robles, J. (2014). Fortalecimiento motivacional para el aprendizaje del inglés a través del uso de recursos educativos digitales abiertos en estudiantes de quinto grado. (Proyecto de grado publicado. Escuela Normal Superior del Distrito de Barranquilla.)

Sánchez, A. (2013). El bilingüismo en los bachilleres colombianos. Cartagena de Indias: Banco de la República.

Touchton, M. (2015). Flipping the classroom and student performance in advanced statistics: Evidence from a quasiexperiment. Journal of Political Science Education, 11, 28-44. doi: 10.1080/15512169.2014.985105

Woolfolk, A. (2010). Educational psychology (13a ed.). New York: Prentice Hall-Pearson.

Cómo citar este artículo (APA):

Ruiz-Robles, J. L. (2016). El efecto del flipped classroom en la motivación por el aprendizaje del inglés como lengua extranjera en estudiantes de nivel preintermedio. AtoZ: novas práticas em informação e conhecimento, 5(2), 104 - 114. Descargado de: http:// dx.doi.org/10.5380/atoz.v5i2.48940 


\section{APÉNDICE A}

\section{Versión adaptada del Attitude/Motivation Test Battery de Gardner (2004).}

Instrucciones. Nos gustaría conocer tu opinión acerca de cada uno de los siguientes enunciados. Tacha la alternativa que más se acerque a tu opinión. Por favor, no dejes preguntas sin contestar. Recuerda que este cuestionario es anónimo; tus respuestas no afectarán tus calificaciones.

\section{Opciones de respuesta:}

Totalmente de acuerdo-5

De acuerdo-4

$\mathrm{Ni}$ en acuerdo ni en desacuerdo-3

En desacuerdo-2

Muy en desacuerdo-1

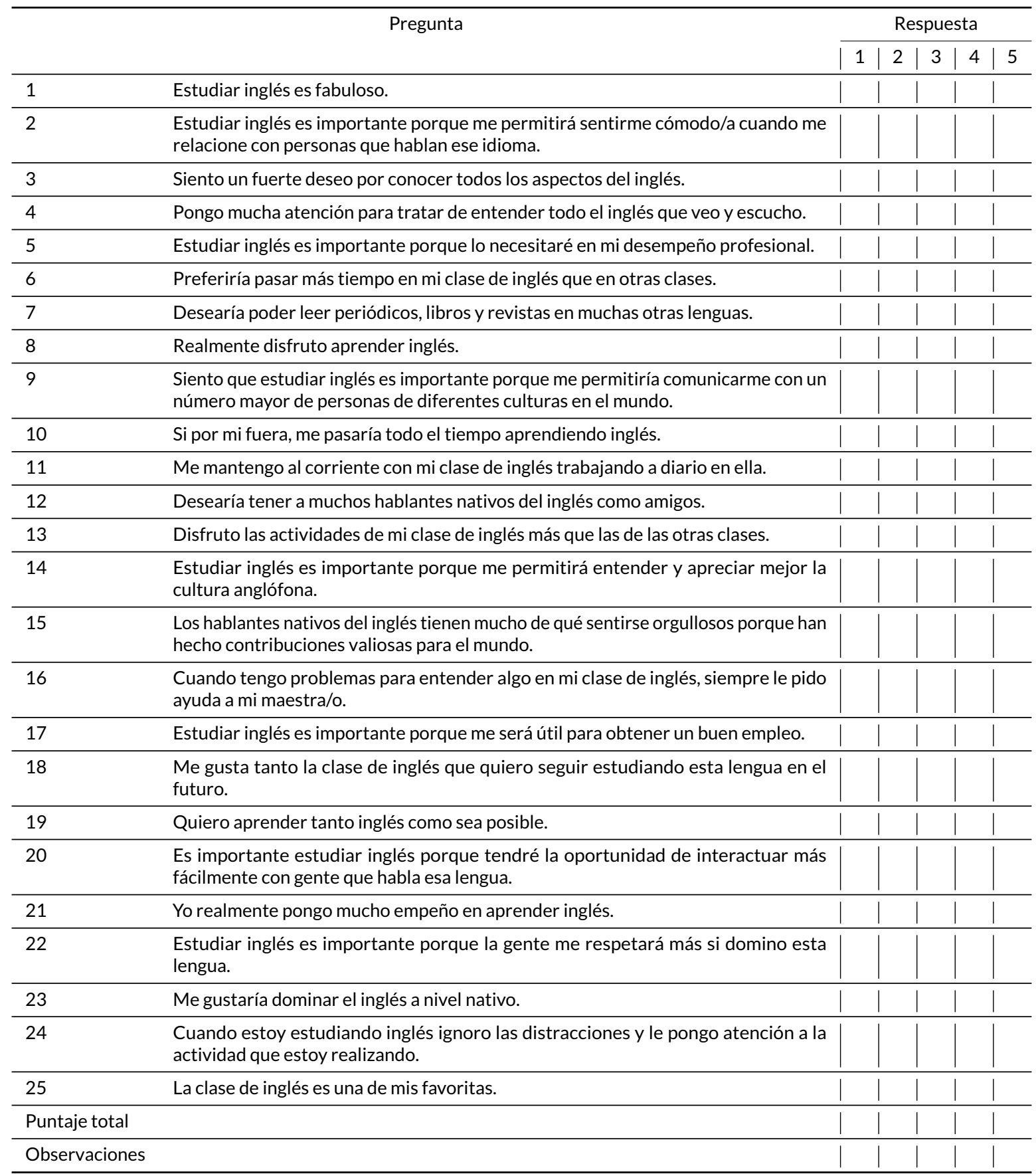


APÉNDICE B - PUNTAJES DEL ATTITUDE/MOTIVATION TEST BATTERY DE GARDNER (2004) ANTES Y DESPUÉS DE LA INTERVENCIÓN.

\begin{tabular}{|c|c|c|c|c|c|}
\hline \multicolumn{3}{|c|}{ Grupo control } & \multicolumn{3}{|c|}{ Grupo experimental } \\
\hline Estudiante & Puntaje pretest & Puntaje postest & Estudiante & Puntaje pretest & Puntaje postest \\
\hline 1 & 35 & 36 & 1 & 36 & 70 \\
\hline 2 & 30 & 33 & 2 & 33 & 65 \\
\hline 3 & 25 & 25 & 3 & 25 & 40 \\
\hline 4 & 80 & 81 & 4 & 76 & 90 \\
\hline 5 & 45 & 43 & 5 & 47 & 64 \\
\hline 6 & 50 & 51 & 6 & 38 & 66 \\
\hline 7 & 52 & 52 & 7 & 48 & 50 \\
\hline 8 & 35 & 37 & 8 & 49 & 85 \\
\hline 9 & 40 & 45 & 9 & 33 & 50 \\
\hline 10 & 100 & 101 & 10 & 25 & 25 \\
\hline 11 & 45 & 44 & 11 & 40 & 70 \\
\hline 12 & 50 & 50 & 12 & 42 & 61 \\
\hline 13 & 37 & 38 & 13 & 36 & 58 \\
\hline 14 & 41 & 42 & 14 & 33 & 52 \\
\hline 15 & 75 & 76 & 15 & 50 & 80 \\
\hline 16 & 45 & 47 & 16 & 81 & 115 \\
\hline 17 & 38 & 38 & 17 & 43 & 69 \\
\hline 18 & 46 & 48 & 18 & 51 & 73 \\
\hline 19 & 50 & 49 & 19 & 52 & 65 \\
\hline 20 & 32 & 33 & 20 & 37 & 59 \\
\hline 21 & 25 & 25 & 21 & 45 & 78 \\
\hline 22 & 39 & 40 & 22 & 36 & 74 \\
\hline 23 & 43 & 42 & 23 & 33 & 59 \\
\hline 24 & 36 & 36 & & & \\
\hline 25 & 47 & 48 & & & \\
\hline
\end{tabular}

